\title{
Artificial Neural Network and Fuzzy Clustering Methods in Segmenting Sputum Color Images for Lung Cancer Diagnosis
}

\author{
Fatma Taher and Rachid Sammouda \\ Department of Computer Engineering, khalifa University, Sharjah, \\ United Arab Emirates \\ Fatma.Taher@kustar.ac.ae \\ Department of Computer science, University of Sharjah, Sharjah, \\ United Arab Emirates \\ Rsammouda@sharjah.ac.ae
}

\begin{abstract}
Lung cancer is cancer that starts in the lungs. Cancer is a disease where cancerous cells grow out of control, taking over normal cells and organs in the body. The early detection of lung cancer is the most effective way to decrease the mortality rate. In this paper we compare two methods, a modified Hopfield Neural Network (HNN) and a Fuzzy C-Mean (FCM) Clustering Algorithm, used in segmenting sputum color images. The segmentation results will be used as a base for a Computer Aided Diagnosis (CAD) system for early detection of lung cancer. Both methods are designed to classify the image of $\mathrm{N}$ pixels among $\mathrm{M}$ classes or regions. Due to intensity variations in the background of the raw images, a pre-segmentation process is developed to standardize the segmentation process. In this study, we used 1000 sputum color images to test both methods, and HNN has shown a better classification result than FCM; however the latter was faster in converging.
\end{abstract}

Keywords: Lung cancer Diagnosis, Sputum Cells, Hopfield Neural Network, Fuzzy C-Mean Clustering .

\section{Introduction}

Lung cancer is considered to be the leading cause of cancer death throughout the world and it is difficult to detect in its early stages because symptoms appear only at advanced stages [1]. Physicians use several techniques to diagnose lung cancer, such as chest radiograph and sputum cytological examination where a sputum sample can be analyzed for the presence of cancerous cells. However, the analysis process of the images obtained by this technique is still suffering from some limitation caused by the scanning process. Recently, some medical researchers have proven that the analysis of sputum cells can lead to a successful diagnosis of lung cancer [2]. For this reason we attempt to come with automatic diagnostic system for detecting the lung cancer in its early stages based on the analysis of the sputum color images. 
In order to formulate a rule we have developed a technique for unsupervised segmentation of the sputum color image. In order to divide the images into several meaningful sub regions, image segmentation has been used as the first step in image analysis, there are many algorithms which have been proposed in other articles for medical image segmentation, such as histogram analysis, regional growth, edge detection and pixel classification. Good reference of this technique can be found in [3]. Other authors have considered the use of the color information as the key discriminate factor for cell segmentation for lung cancer diagnosis The analysis of sputum images have been used in [4] for detecting tuberculosis; it consists of analyzing sputum images for detecting bacilli. In this paper, two basic techniques have been applied: Hopfield Neural Network (HNN) and Fuzzy C-Mean Clustering Algorithm (FCM) to segment sputum color images prepared by the standard staining method described in [5]. In computer memory, each image is represented as three separate pixel matrices corresponding to red, green and blue intensity $(R, G$, and $B$ image planes). The segmentation is performed based on the result of a preprocessing for the extraction of region of interest (ROI). We present result of the segmentation of some images for both methods. The remanding sections of this paper is organized as follows. In Section 2, Hopfield Neural Network (HNN) segmentation algorithm is introduced. In Section3, fuzzy clustering algorithm is described. In Section 4, segmentation result and discussion are presented. Finally in Section 5, the conclusion is drawn and several issues for future works are discussed.

\section{Hopfield Neural Network}

Hopfield Neural Network (HNN) is one of the artificial neural networks, which has been proposed for segmenting both gray-level and color images. In [6], the authors present the segmentation problem for gray-level images as minimizing a suitable energy function with $\mathrm{HNN}$; it derived the network architecture from the energy function. The existing algorithm for color image segmentation can be found in [7], where the authors present an algorithm for segmentation of medical stained images where the nuclei cell and background were classified into three classes, and the input was the RGB component of the used images. In our work we used the Hopfield neural network HNN algorithm as our segmentation method. The HNN is very sensitive to intensity variation and it can detect the overlapping cytoplasm classes. HNN is considered as unsupervised learning therefore; the network classifies the feature space without teacher based on the compactness of each cluster calculated using the Euclidean distance measure between the kth pixel and the centroid of class 1 . The neural network structure consists of a grid of $\mathrm{N} \times \mathrm{M}$ neurons with each column representing a cluster and each row representing a pixel. The network is designed to classify the image of $\mathrm{N}$ pixels of $\mathrm{P}$ features among $\mathrm{M}$ classes, such that the assignment of the pixels minimizes the criterion function:

$$
E=\frac{1}{2} \sum_{k=1}^{N} \sum_{l=1}^{M} R_{k l}^{2} V_{k l}^{2}
$$


where $\mathrm{R}_{\mathrm{kl}}$ is considered as the Euclidean distance measure between the kth pixel and the centroid of class $1, \mathrm{Vkl}$ is the output of the kth neurons. The minimization is achieved using HNN and by solving the motion equations satisfying:

$$
\frac{\partial u_{i}}{\partial t}=-\mu(t) \frac{\partial E}{\partial V_{i}}
$$

where $\mu(t)$ is as defined in [6] a scalar positive function of time used to increase the convergence speed of the HNN. By applying the relation (2) to equation (1), we get a set of neural dynamics given by:

$$
\frac{d U_{k l}}{d t}=-\mu(t)\left[R_{k l}^{2} V_{k l}\right]
$$

Where $\mathrm{U}_{\mathrm{kl}}$ and $\mathrm{V}_{\mathrm{kl}}$ are the input and output of the $\mathrm{k}_{\mathrm{th}}$ neuron respectively. To assign a label $\mathrm{m}$ to the $\mathrm{k}_{\mathrm{th}}$ pixel we use the input-output function given by:

$$
\mathrm{v}(\mathrm{t}+1)
$$

The HNN segmentation algorithm can be summarized in the following steps:

1. Initialize the input of neurons to random values.

2. Apply the input-output relation given in (4) to obtain the new output value for each neuron, establishing the assignment of pixel to classes.

3. Compute the centroid for each class

4. Solve the set of differential equation in (3) to update the input of each neuron.

5. Repeat from step 2 until convergence then terminate.

We applied the HNN with the specification mentioned above to one thousand sputum color images and maintain the result for further processing in the following steps. Our algorithm could segment $97 \%$ of the images successfully in nuclei, cytoplasm regions and clear background. Furthermore, HNN takes short time to achieve the desired results. By experiment, HNN needs less than 120 iterations to reach the desired segmentation result in 36 seconds.

\section{Fuzzy Clustering}

Clustering is the process of dividing the data into groups based on similarity of objects; information that is logically similar physically is stored together, in order to increase the efficiency in the database system and to minimize the number of disk access. Let $\left\{\mathrm{X}^{(q)}: \mathrm{q}=1, \ldots \ldots, \mathrm{Q}\right\}$ be a set of $\mathrm{Q}$ feature vectors. Each feature vector $\mathrm{X}^{(q)}=\left(\mathrm{X}^{1}, \ldots, \mathrm{X}^{N}\right)$ has $\mathrm{N}$ components. The process of clustering is to assign the $\mathrm{Q}$ feature vectors into $\mathrm{K}$ clusters $\left\{C^{(k)}: k=1, \ldots, K\right\}$ for each $\mathrm{K}^{\text {th }}$ cluster $\mathrm{C}^{k}$ its center and its designated by $\mathrm{C}^{(k)}$. 
Fuzzy Clustering has been used in many fields like pattern recognition and Fuzzy identification. A variety of Fuzzy clustering methods has been proposed and most of them are based upon distance criteria. The most widely used algorithm is the Fuzzy C-Mean algorithm (FCM), it uses reciprocal distance to compute fuzzy weights. The FCM was introduced by J.C.Bezdek [8]. This algorithm has as input a predefined number of clusters, which is the $\mathrm{k}$ from its name. Means stands for an average location of all the members of particular cluster and the output is a partitioning of $\mathrm{k}$ cluster on a set of objects.

The objective of the FCM cluster is to minimize the total weighted mean square error.

$$
\mathrm{J}=\left(\mathrm{W}^{q k}, \mathrm{C}^{(k)}\right)=\sum_{(q=1, Q} \sum_{(k=1, K)}\left(W_{q k}\right)^{p}\left\|x^{(q)}-c^{(k)}\right\|^{2}
$$

The FCM allows each feature vector to belong to multiple clusters with various fuzzy membership values [9]. Then the final classification will be according to the maximum weight of the feature vector over all clusters. The detail algorithm:

Input: Vectors of objects, each object represent s dimensions, where:

$v=\left\{v_{1}, v_{2}, \ldots \ldots, v_{n}\right\}$. In our case it will be an image pixel; each pixel has 3 dimensions, RGB

$\mathrm{K}=$ number of cluster.

Output: A set of k cluster which minimize sum of distance error.

Algorithm steps:

1. Initialize random weight for each pixel, it uses fuzzy weighting with positive weights $\left\{\mathrm{W}^{q k}\right\}$ between $[0,1]$.

2. Standardize the initial weights for each $\mathrm{q}^{\text {th }}$ feature vector over all $\mathrm{K}$ clusters via

3. Standardize the weights over $\mathrm{k}=1, \ldots, \mathrm{K}$ for each $\mathrm{q}$ to obtain $\mathrm{W}^{q k}$.

4. Compute new centroids $\mathrm{C}^{(k)}, \mathrm{k}=1, \ldots, \mathrm{K} \quad$ via

5. Update the weights $\left\{\mathrm{W}^{q k}\right\}$ via

6. Assign each pixel to a cluster based on the maximum weight.

We applied the FCM clustering algorithm with the specification mentioned above to one thousand sputum color images and maintain the result for further processing in the following steps. Our algorithm segment the images in nuclei, cytoplasm regions and clear background, however, the FCM is not sensitive to intensity variation, therefore, the cytoplasm regions are detected as one cluster when we fixed the cluster number to three, four, five and six. Moreover, FCM failed in detecting the nuclei, it detected only part of it. By experiment, the FCM algorithm takes less than 50 iterations to reach the desired results in 10 seconds on average. 


\section{Segmentation Result and Discussion}

Here, we present the result obtained with two sample images; the first sample containing red cells surrounded by a lot of debris nuclei and a background reflecting a large number of intensity variation in its pixel values as shown in Figure1 (a), and the second sample is composed of blue stained cells shown in Figure 3 (a), Figure 1 (b) and (c) show the segmentation results using HNN and the FCM with the RGB components of the raw image Figure 1 (a), respectively. As is seen in the segmentation result of both algorithms (b) and (c), the nuclei of the cells were not detected, in the case of HNN, and were not accurately represented in (c). For this reason we developed a mask to extract our regions of interest, described in [6], and the result is shown in Figure $1(\mathrm{~d})$.

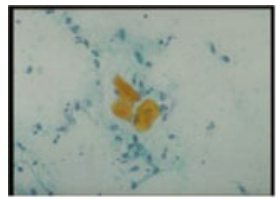

(a)

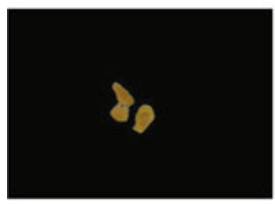

(d)

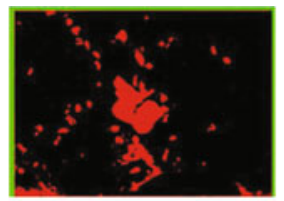

(b)

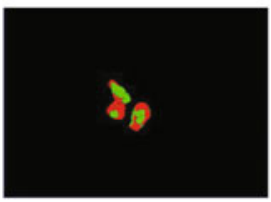

(e)

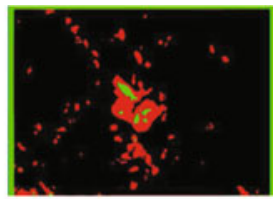

(c)

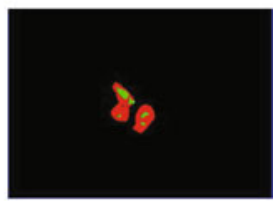

(f)

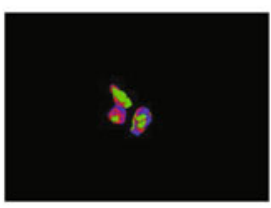

(g)

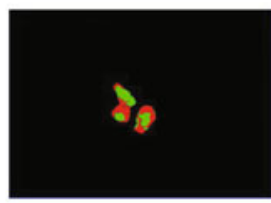

(h)

Fig. 1. (a) Sample of sputum color image stained with blue and red dyes. (b) and (c) The segmentation result using HNN and the FCM, respectively, with the RGB components of the raw image. (d) The result of pre-segmentation masking process. (e) and (f) show the segmentation result using HNN and FCM with the RGB components of (d)by fixing the clusters number to three, respectively, $(\mathrm{g})$ and $(\mathrm{h})$ are the results obtained by fixing the clusters number to four.

Figure 1 (e) and (f) show the segmentation result using HNN and FCM with the RGB components of (d). By fixing the cluster numbers to three, respectively, we realized that in the case of $\mathrm{HNN}$, the nuclei were detected but not precisely. And in 
the case of the FCM only part of the nuclei has been detected. We increased the cluster numbers to four as an attempt to solve the nuclei detection problem. The results are shown in Figure $1(\mathrm{~g})$ and $(\mathrm{h})$ for both HNN and FCM, respectively.

Comparing the HNN segmentation result in (g) to the raw image (d), we can say that the nuclei regions were detected perfectly, and also their corresponding cytoplasm regions. However, due to the problem of intensity variation in the raw image (d) and also due to the sensitivity of HNN, the cytoplasm regions were represented by two clusters. These cytoplasm clusters may be merged later if the difference in their mean values is not large.

Comparing the FCM segmentation result in (h) to the raw image (d), the nuclei regions are detected but they present a little overlapping in the way that the two different nuclei may be seen or considered as one nucleus, and this can affect the diagnosis results. The cytoplasm regions are smoother than in the case of HNN, reflecting that the FCM is less sensitive to the intensity variation than HNN.

A quantitative reflection of the above comparison and discussion is shown in Figure 2, where it can be seen that the segmentation error at convergence is smaller with HNN than with the FCM. However the FCM converges fifty iterations earlier than HNN.

Figure 3 (a) shows a sample of sputum color image stained with blue dye, (b) and (c) show the segmentation results using $\mathrm{HNN}$ and the FCM with the RGB components of the raw image (a), respectively. As is seen in the segmentation results of both algorithms in (b) and (c) the nuclei have not been detected and the background presents a lot of intensity variation. A filter was needed to minimize the affect of the intensity variation in the raw image as described in [6].

The result of this filter is shown in (d); (e) and (f) are the segmentation results obtained using HNN and FCM with RGB components of (d) with four clusters. Here, the nuclei have been detected; however a color cluster is missing in the result of FCM (f). The same applies for the previous case of the red cells; HNN is more sensitive to intensity variation between nuclei-nuclei or nuclei-cytoplasm regions. This is clear in Figure 4 which shows quantitatively, the energy function of HNN and FCM during the segmentation process of the blue sample.

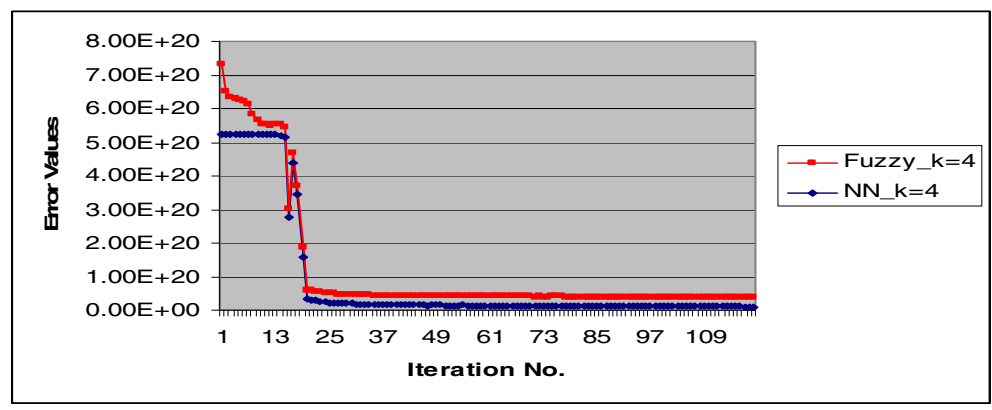

Fig. 2. Curves of the HNN and the FCM energy functions during the segmentation process 


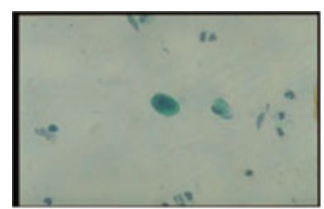

(a)

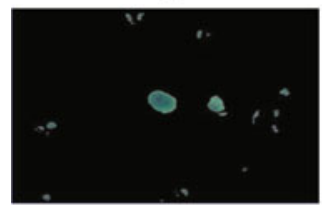

(d)

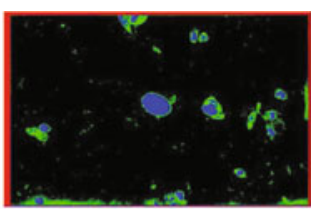

(b)

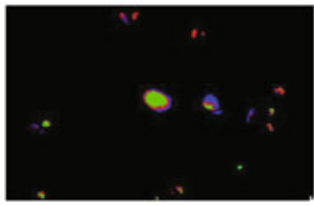

(e)

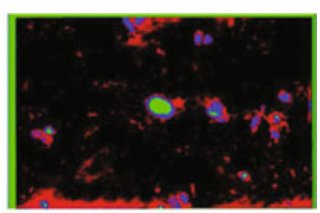

(c)

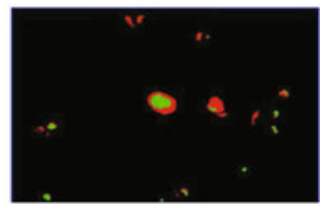

(f)

Fig. 3. (a) A sample of sputum color image stained with blue dyes, (b) and (c) the segmentation result using HNN and the FCM with the RGB components of the raw image(a), respectively. (d) The result of pre-segmentation masking process. (e) and (f) The segmentation result using HNN and FCM with the RGB components of (d) by fixing the clusters number to four, respectively.

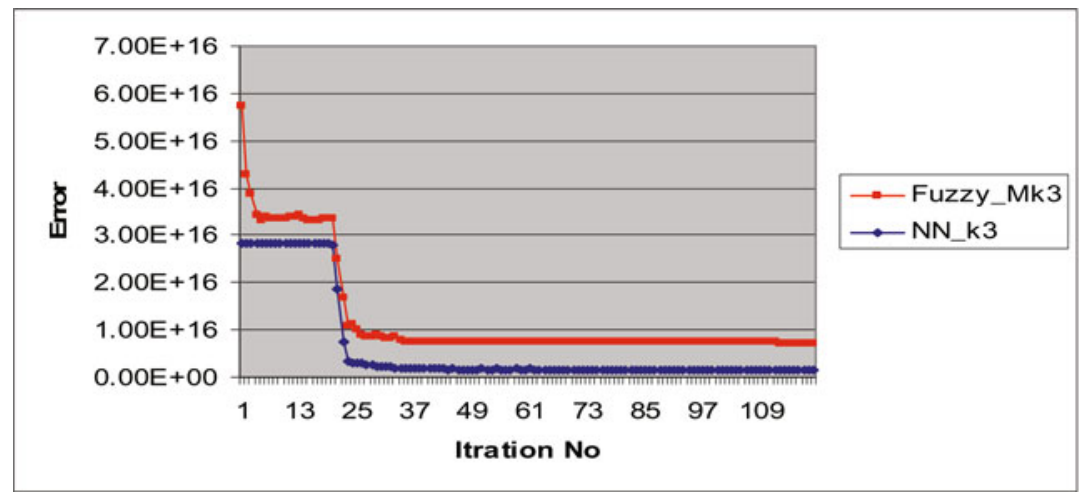

Fig. 4. Curve of the HNN and the FCM energy functions during the segmentation process

\section{Conclusion}

In this study, we present two methods for the segmentation of sputum cell color images, which will be used as a basis for a Computer Aided Diagnosis (CAD) system for early detection of lung cancer.

The presented methods, use a pre-classification algorithm to extract the regions of interest with the raw color image component in the RGB color space, and remove the debris cells. As shown in the previous section, HNN has shown a better classification result than a FCM clustering algorithm, however the latter was faster in convergence. In the future, we plan to consider a Bayesian decision theory for the detection of the lung cancer cells as soon as a more extended dataset is available. 


\section{References}

1. The National Women's Health Information Center, U.S Department of Health and Human Services office on Women's Health, Lung Cancer (2003), http: / / www . 4woman.gov/faq/lung.htm

2. Herb, F.: HuilongGuan Culture, Lung cancer (2002), http: / / www. 4uherb.com/cancer/lung/about.htm

3. Lin, J.S., Chenz, K.S., Mao, C.-W.: A Fuzzy Hopfield Neural for Medical Image Segmentation. IEEE Trans. Nuclear Science 43(4) (August 1996)

4. Forero-Vargas, M., Sierra-Ballen, E.L., Alvarez-Borrego, J., Pacheco, J.L.P., Cristobal, G., Alcala, L., Desco, M.: Automatic Sputum Color Image Segmentation for Tuberculosis Diagnosis. In: Proc. SPIE, pp. 251-261 (2001)

5. Sammouda, R., Niki, N., Nishitani, H., Nakamura, S., Mori, S.: Segmentation of Sputum Color Image for Lung Cancer Diagnosis. In: Proceeding of International Conference on image Analysis and Processing, Italy, September 1997, p. 243 (1997)

6. Sammouda, R., Niki, N., Nishitani, H., Kyokage, E.: Segmentation of Sputum Color Image for Lung Cancer Diagnosis based on Neural Network. Journal of IEICE Transaction on information and systems E81_D(8), 862-871 (1998)

7. Taher, F., Sammouda, R.: Identification of Lung Cancer based on shape and Color. In: Proceeding of the 4th International Conference on Innovation in Information Technology, Dubai, UAE, November 2007, pp. 481-485 (2007)

8. Bezdek, J.C.: Fuzzy Mathematics in Pattern Classification, Ph.D. thesis, Center for Applied Mathematics, Cornell University, Ithica, N.Y (1973)

9. Chen, Y.-S.: An Efficient Fuzzy c-means Clustering Algorithm for Image Data. In: Mathematical problems in Engineering and Aerospace Science (2004) 